The choice of antibiotic for surgical prophylaxis was considered to be appropriate in 93/144 (64.6\%) patients pre-guideline and $142 / 171(83 \%)$ post guideline. The duration of prophylaxis was considered appropriate in $77 / 144(53.5 \%)$ procedures pre and 103/171 (60\%) procedures post guideline. The number of single prophylactic doses prescribed was 32/144 (22.2\%) prior to the guidelines compared with 48/171 (28.1\%) following guideline introduction.

Conclusion An organisation wide antibiotic surgical prophylaxis guideline can have a positive impact on practice. The availability of the guideline has improved choice of antibiotic, duration and over all documentation. Use of multiple doses can be partially explained by complex surgical patients however further work is required to explore the reasons for prolonged surgical prophylaxis in paediatrics as well as optimisation of the choice of therapy. Whilst the evidence for paediatric antibiotic surgical prophylaxis is limited ${ }^{1}$ this study demonstrated that an improvement in antimicrobial stewardship against national guidance $^{2}$ can be achieved.

\section{P38 AN IMPACT ANALYSIS OF A PAEDIATRIC ANTIBIOTIC SURGICAL PROPHYLAXIS GUIDELINE}

Hirminder Ubhi, Jeff Aston, Mitul Patel. Birmingham Children's Hospital

\subsection{6/archdischild-2015-308634.46}

Aim The aim of this study was to evaluate the impact of an organisation wide paediatric antibiotic surgical prophylaxis guideline.

Method A before and after study was conducted during the period Jun to Aug 2012 (pre-guideline implementation) and Jun to Dec 2013 (post-guideline implementation) on six surgical wards (two general surgery wards, neurosurgery, urology, intensive care and neonatal surgery). A random sample of ten patients per month were identified by ward pharmacists. The patients' drug charts, operation records and medical notes were reviewed and data collected using a standardised data collection tool. Data were collected on the patients' procedure undertaken, prescribed surgical prophylactic antibiotic(s), antibiotic administration time, the number of doses administered and whether an indication for use and duration was specified when the antibiotic was prescribed. The data were reviewed by an antimicrobial pharmacist and medical microbiologist to determine appropriateness of therapy. The data were collated using Microsoft Excel and analysed using descriptive statistics.

Results One hundred and forty four patients were reviewed prior to implementation of the guidelines and 171 patients following guideline introduction.

Prior to the introduction of the guidelines, an antibiotic indication and duration, respectively, was documented in 93/144 $(64.6 \%)$ and $77 / 144(53.5 \%)$ procedures compared with $142 /$ $171(83 \%)$ and 102/171 (59.6\%) post guideline. The time of antibiotic administration was recorded in 109/144 (75.7\%) procedures pre-guideline and 167/171 (97.7\%) post guideline. Where the time of administration was recorded, in 104/109 (95.4\%) procedures, antibiotics were administered within 60 minutes of incision pre-guideline compared with 126/167 (75.4\%) post guideline.

\section{REFERENCES}

1 Bratzler DW, Dellinger EP, Olsen KM. Clinical Practice Guidelines for Antimicrobial Prophylaxis in Surgery. Am J Health-Syst Pharm 2013;70:195-283.

2 Department of Health. Advisory Committee on Antimicrobial Resistance and Healthcare Associated Infection (ARHAI), 2011. Antimicrobial Stewardship: "Start Smart then - Then Focus" Guidance for antimicrobial stewardship in hospitals (England). https://www.gov.uk/government/uploads/system/uploads/attachment_data/ file/215308/dh_131181.pdf (accessed 19 May 2014). 